\title{
Error Estimates for Orthogonal Matching Pursuit and Random Dictionaries
}

\author{
Paweł Bechler · Przemysław Wojtaszczyk
}

Received: 10 August 2009 / Revised: 10 May 2010 / Accepted: 10 June 2010 /

Published online: 26 October 2010

(C) The Author(s) 2010. This article is published with open access at Springerlink.com

\begin{abstract}
In this paper we investigate the efficiency of the Orthogonal Matching Pursuit algorithm (OMP) for random dictionaries. We concentrate on dictionaries satisfying the Restricted Isometry Property. We also introduce a stronger Homogenous Restricted Isometry Property which we show is satisfied with overwhelming probability for random dictionaries used in compressed sensing. For these dictionaries we obtain upper estimates for the error of approximation by OMP in terms of the error of the best n-term approximation (Lebesgue-type inequalities). We also present and discuss some open problems about OMP. This is a development of recent results obtained by D.L. Donoho, M. Elad and V.N. Temlyakov.
\end{abstract}

Keywords Orthogonal matching pursuit $\cdot$ Coherence $\cdot$ Restricted isometry property $\cdot$ Random dictionaries $\cdot$ Lebesgue inequalities $\cdot$ Nonlinear approximation

Mathematics Subject Classification 41A25 (15A52, 41A17, 41A46)

\section{Introduction}

In this paper we investigate the efficiency of the Orthogonal Matching Pursuit algorithm (OMP), also known in literature as the Orthogonal Greedy Algorithm, for random dictionaries. OMP (cf. $[9,10]$ ) is a well-known greedy algorithm widely

Communicated by Vladimir N. Temlyakov.

This research was partially supported by the Polish Ministry of Science and Higher Education grant no. N N201 269335.

P. Bechler $(\bowtie) \cdot$ P. Wojtaszczyk

University of Warsaw, ul. Banacha 2, 02-097 Warszawa, Poland

e-mail: pbechler@mimuw.edu.pl

P. Wojtaszczyk

e-mail: wojtaszczyk@mimuw.edu.pl 
used in approximation theory, statistical estimations, and compressed sensing (for a general review of greedy algorithms see [13]). One of its main features is that it can be used with an arbitrary dictionary. However, the efficiency of the algorithm seems to depend very strongly on the properties of the dictionary.

In this paper we work in the context of a Hilbert space $\mathcal{H}$ (which may be assumed to be finite dimensional) with the scalar product $\langle$,$\rangle and the norm \|\|$. The dictionary is a subset $\Phi=\left\{\phi_{j}: j \in J\right\} \subset \mathcal{H}$ such that $\overline{\operatorname{span} \Phi}=\mathcal{H}$. We usually assume that $\|x\|$ is close to 1 for $x \in \Phi$. Usually in the literature it is assumed that $\|x\|=1$ for $x \in \Phi$ (see, e.g., [13]). However, for random dictionaries it is very rarely satisfied. On the other hand, for such dictionaries $\|x\|$ is close to 1 with great probability.

In the space $\mathcal{H}$ we consider the Orthogonal Matching Pursuit algorithm with respect to the dictionary $\Phi$. This algorithm obtains iteratively a sequence $\mathrm{OMP}_{n} f \in \mathcal{H}$ of approximants of a given element $f \in \mathcal{H}$ in the following way:

- Define $\mathrm{OMP}_{0} f=0$.

- Given $\mathrm{OMP}_{n-1} f$ choose $j_{n} \in J$ such that

$$
\left|\left\langle f-\mathrm{OMP}_{n-1} f, \phi_{j_{n}}\right\rangle\right|=\sup \left\{\left|\left\langle f-\mathrm{OMP}_{n-1} f, \phi_{j}\right\rangle\right|: j \in J\right\}
$$

and define $\mathrm{OMP}_{n} f$ as the orthogonal projection of $f$ onto the subspace $\operatorname{span}\left\{\phi_{j_{1}}, \ldots, \phi_{j_{n}}\right\}$.

For a fixed $f \in H$ we denote $f_{n}=f-\mathrm{OMP}_{n} f$.

The standard measure of approximation power of a dictionary is the error of the best $m$-term approximation. We define the set of $m$-sparse vectors (with respect to the dictionary $\Phi)$ as

$$
\Sigma_{m}(\Phi)=\Sigma_{m}=\left\{\sum_{j=1}^{m} a_{j} \phi_{j} \in \mathcal{H}:\left\{\phi_{j}\right\}_{j=1}^{m} \subset \Phi\right\} .
$$

For a given $f \in \mathcal{H}$ we define its error of the best $m$-term approximation (cf. [13]) as

$$
\sigma_{m}(f, \Phi)=\inf \left\{\|f-z\|: z \in \Sigma_{m}\right\}
$$

Clearly, we always have $\sigma_{m}(f) \leq\left\|f-\mathrm{OMP}_{m}(f)\right\|=\left\|f_{m}\right\|$.

When our dictionary is an orthonormal basis then, obviously, $\sigma_{m}(f)=$ $\left\|f-\mathrm{OMP}_{m}(f)\right\|$ for each $f \in \mathcal{H}$. Unfortunately, this does not hold for most dictionaries. The fundamental and still largely unanswered question is how close $\mathrm{OMP}_{m} f$ can get to this optimal rate of approximation given by $\sigma_{m}(f)$. It is to be expected that the answer to the above question must depend on some auxillary properties of the dictionary. We will discuss it in more detail in the last section of this paper.

We concentrate on a random dictionary in $\mathbb{R}^{n}$ of the following form: $\Phi=$ $\left\{\phi_{1}, \ldots, \phi_{N}\right\}$, with $\phi_{j}=\frac{1}{\sqrt{n}}\left(\eta_{1, j}, \ldots, \eta_{n, j}\right)$ where $\left(\eta_{i, j}\right)_{i=1}^{n} \underset{j=1}{N}$ are independent, identically distributed, mean zero subgaussian random variables with $\mathbb{E} \eta_{i, j}^{2}=1$. It is a natural class of dictionaries which recently gained prominence due to its importance in compressed sensing (see, e.g., [2, 4, 6]). In compressed sensing we think about such a dictionary as a matrix whose columns are $\phi_{j}$ 's. Any approximation 
scheme for such a dictionary provides a decoder for a measurement matrix $\Phi$. For such random dictionaries we prove that there exist positive constants $c, c_{1}, c_{2}$ such that for $K=c n / \log _{2} N$ and $0 \leq k<S \leq K$ we have

$$
\left\|f_{S}\right\|^{2} \leq c_{1}\left\|f_{k}\right\|\left(\sigma_{S-k}\left(f_{k}\right)+c_{2} \sqrt{S / K}\left\lceil\log _{2}(2 S-k)\right\rceil\left\|f_{k}\right\|\right) .
$$

As the main application we derive the estimate

$$
\left\|f_{\left\lceil m\left(4 \log _{2} m-1\right)\right\rceil}\right\| \leq c \sigma_{m}(f),
$$

which is valid for $m \leq c \sqrt{K}$. These results improve for random dictionaries the results from [7]. Technically speaking, the results in [7] are for dictionaries having small coherence, while we introduce a different assumption: Homogenous Restricted Isometry Property.

\section{Dictionaries}

Despite the fact that we are mostly interested in random dictionaries, our main results are formally deterministic. We isolate the properties which a random dictionary has with overwhelming probability and prove our results under the assumption that our dictionary has these properties. A widely used characteristic of a dictionary is its coherence.

Definition 1 The coherence of a dictionary $\Phi$ is defined as

$$
\eta=\eta(\Phi)=\sup \left\{\left|\left\langle\phi_{1}, \phi_{2}\right\rangle\right|: \phi_{1}, \phi_{2} \in \Phi, \phi_{1} \neq \phi_{2}\right\} .
$$

Recently, especially in the context of compressed sensing, a property called the Restricted Isometry Property (RIP for short) became very useful. Another analysis of OMP under the RIP assumption, mostly motivated by compressed sensing, is the recent work [5] by M.A. Davenport and M.B. Wakin. There is no overlap between our results and [5]. The main difference between our approach and theirs is that they require $\operatorname{RIP}(K, \varepsilon)$ for a very small $\varepsilon$ dependent on $K$.

Let us recall the following well-known definition (c.f. [2]) phrased in terms of the dictionary instead of the measurement matrix:

Definition 2 The dictionary $\Phi$ satisfies the Restricted Isometry Property $\operatorname{RIP}(K, \varepsilon)$, with $0<\varepsilon<1$, if for any subset $I \subset J$ with $\# I \leq K$ and any scalars $a_{j}, j \in I$, the following inequalities hold:

$$
(1-\varepsilon)\left(\sum_{j \in I}\left|a_{j}\right|^{2}\right)^{1 / 2} \leq\left\|\sum_{j \in I} a_{j} \phi_{j}\right\| \leq(1+\varepsilon)\left(\sum_{j \in I}\left|a_{j}\right|^{2}\right)^{1 / 2} .
$$

This definition in particular means that $\left\{\phi_{j}\right\}_{j \in I}$ is a Riesz basis for its linear span. From [3, Proposition 3.6.4] we get the following: 
Proposition 2.1 If the dictionary $\Phi$ satisfies $\operatorname{RIP}(K, \varepsilon)$ and $I \subset J$ such that $\# I \leq K$ and $f \in \operatorname{span}\left\{\phi_{i}: i \in I\right\}$, then

$$
(1-\varepsilon)\|f\| \leq\left(\sum_{i=1}^{n}\left|\left\langle f, \phi_{i}\right\rangle\right|^{2}\right)^{1 / 2} \leq(1+\varepsilon)\|f\| .
$$

The relation between RIP and coherence is expressed in the following proposition:

Proposition 2.2 The following statements are true:

(i) If the dictionary $\Phi$ has coherence $\eta=\eta(\Phi)$, then it has $\operatorname{RIP}(K, \eta(K-1))$ for $K \leq \eta^{-1}+1$

(ii) If the dictionary $\Phi$ satisfies $\operatorname{RIP}(K, \varepsilon)$, then $\eta(\Phi) \leq \varepsilon(2+\varepsilon)$.

Proof (i) is shown in [7, Lemma 2.1] while (ii) is obtained by straightforward calculation.

Let us introduce the following:

Definition 3 The dictionary $\Phi$ has Homogenous Restricted Isometry Property $\operatorname{HRIP}(k, \delta), 0<\delta<1$, if for any set $T \subset\{1, \ldots, N\}$ with $\# T=l \leq k$ and any sequence of numbers $a_{j}$ we have

$$
\left(1-\delta \sqrt{\frac{l}{k}}\right)\left(\sum_{j \in T}\left|a_{j}\right|^{2}\right)^{1 / 2} \leq\left\|\sum_{j \in T} a_{j} \phi_{j}\right\| \leq\left(1+\delta \sqrt{\frac{l}{k}}\right)\left(\sum_{j \in T}\left|a_{j}\right|^{2}\right)^{1 / 2} .
$$

The following theorem justifies this definition:

Theorem 2.3 Suppose that integers $n, N$ and numbers $0<\delta<1$ and $a>0$ are given and the dictionary $\Phi=\left\{\phi_{1}, \ldots, \phi_{N}\right\} \subset \mathbb{R}^{n}$ is as described above. Then there exist constants $c>0$ which depend only on the subgaussian distribution involved, $\delta$ and a such that the dictionary $\Phi$ satisfies $\operatorname{HRIP}(k, \delta)$ for $k=\lfloor c n / \log N\rfloor$ with probability $\geq 1-3 N^{-a}$.

Proof The proof relies on standard arguments. It is known, see, e.g., [12], that such matrices (dictionaries) satisfy the concentration of measure property of the form: there is $c_{0}>0$ such that for each $1 \geq \epsilon>0$ for any $x \in \mathbb{R}^{N}$ we have

$$
\mathbb{P}\left(\left\|\left|\sum_{j=1}^{N} x_{j} \phi_{j}\left\|^{2}-\right\| x\left\|^{2} \mid>\epsilon\right\| x \|^{2}\right) \leq 2 e^{-n c_{0} \epsilon^{2}} .\right.\right.
$$

Then Lemma 5.1 from [1] says that for any fixed set $T \subset\{1, \ldots, N\}$ with \#T=l the inequality $(2.1)$ fails with probability $\leq 2(12 / \delta)^{l} e^{-c_{0}(\delta / 2)^{2} n}$. Since there are $\left(\begin{array}{c}N \\ l\end{array}\right)<$ $(e n / l)^{l}$ such subsets we see that (2.1) fails for all sets $T$ with \#T=l with probability

$$
\leq 2\left(\frac{e N}{l}\right)^{l}\left(\frac{12}{\delta}\right)^{l} e^{-c_{0} \delta^{2} n / 4}
$$


so (2.2) fails for all sets $T$ with \#T=l with probability

$$
\begin{aligned}
& \leq 2\left(\frac{e N}{l}\right)^{l}\left(\frac{12 \sqrt{k}}{\delta \sqrt{l}}\right)^{l} e^{-c_{0} \delta^{2} \ln /(4 k)} \\
& =2 \exp \left[l\left(\ln e N+\ln 12+\ln (1 / \delta)+\frac{1}{2} \ln (k / l)\right)-l \ln l-c_{0} \delta^{2} \frac{\ln }{4 k}\right] \\
& \leq 2 \exp \left(\gamma l \ln N-c_{0} \delta^{2} \frac{\ln }{4 k}\right)
\end{aligned}
$$

where $\gamma>0$ is a constant depending on $\delta$. Now we set

$$
k=\left\lfloor\frac{c_{0} \delta^{2}}{\gamma \mu} \cdot \frac{n}{\ln N}\right\rfloor,
$$

where $\mu=4(1+a / \gamma)$. We continue our estimates to get

$$
\leq 2 \exp \left(\gamma\left(1-\frac{\mu}{4}\right) l \ln N\right)=2 \exp -a l \ln N=2 N^{-a l} .
$$

Summing over $l=1,2, \ldots$ we get that $\operatorname{HRIP}(k, \delta)$ fails with probability at most $2 \sum_{l=1}^{\infty} N^{-a l} \leq \frac{2}{N^{a}-1}$, which implies the theorem.

\section{Main Results}

We prove the following theorem, which is an RIP analogue of Theorem 1.3 from [7]:

Theorem 3.1 Assume that the dictionary $\Phi$ satisfies $\operatorname{RIP}(2 S, \varepsilon)$ and $0 \leq k<S$. Then

$$
\left\|f_{S}\right\|^{2} \leq 2\left\|f_{k}\right\|\left(\sigma_{S-k}\left(f_{k}\right)+4 \varepsilon\left(2+\left\lceil\log _{2} S\right\rceil\right)\left\|f_{k}\right\|\right) .
$$

Note in particular that seting $k=0$ gives us

$$
\left\|f_{S}\right\|^{2} \leq C\|f\|\left(\sigma_{S}(f)+A \epsilon\|f\|\right) .
$$

We observe that while a connection between coherence and RIP exists, as Proposition 2.2 indicates, it seems that neither Theorem 3.1 follows from a similar inequality shown in [7], nor vice versa.

To prove this theorem we require the following fact:

Proposition 3.2 Let $0<\varepsilon<1$ and $A=\left[a_{i, j}\right]$ be an $n \times n$ upper triangular matrix such that for any $x \in \mathbb{R}^{n}$,

$$
(1-\varepsilon)\|x\| \leq\|A x\| \leq(1+\varepsilon)\|x\|
$$

and $\left|a_{i, i}\right| \geq 1-\varepsilon$ for $i=1, \ldots, n$. Let $i_{1}, i_{2}, \ldots, i_{n} \in\{0,1, \ldots, n\}$ be such that

$$
i_{j}<j \quad \text { for } j=1, \ldots, n \text { and } i_{j+1} \geq i_{j} .
$$


Let $B=\left[b_{i, j}\right]$ be an $n \times n$ matrix defined by

$$
b_{i, j}= \begin{cases}a_{i, j} & \text { if } 1 \leq i \leq i_{j} \\ 0 & \text { otherwise }\end{cases}
$$

Then $\|B\| \leq 4 \varepsilon\left\lceil\log _{2} n\right\rceil$.

The idea of the proof is to cut the matrix $B$ into rectangular pieces. In this we follow [11]. The heart of the proof of Proposition 3.2 is the following lemma:

Lemma 3.3 Let $A$ be an $n \times n$ matrix as in Proposition 3.2. Let $1<r<n$ and $A_{1}$ and $A_{2}$ be respectively $r \times r$ and $(n-r) \times(n-r)$ upper diagonal matrices such that

$$
A=\left[\begin{array}{cc}
A_{1} & C \\
0 & A_{2}
\end{array}\right] .
$$

Then $A_{1}$ and $A_{2}$ satisfy (3.2) and $\|C\| \leq 4 \varepsilon$.

Proof For $y \in \mathbb{R}^{r}$ and $x=\left[\begin{array}{l}y \\ 0\end{array}\right] \in \mathbb{R}^{n}$ we have $\|A x\|=\left\|A_{1} y\right\|$. Hence, for any $y \in \mathbb{R}^{r}$ the matrix $A_{1}$ satisfies

$$
(1-\varepsilon)\|y\| \leq\left\|A_{1} y\right\| \leq(1+\varepsilon)\|y\| .
$$

Because the inequality (3.2) is also satisfied if $A$ is replaced by $A^{H}$, analogous argument gives that the same estimates hold for $A_{2}$.

We now estimate $\|C\|$. Clearly $\|C\| \leq\|A\|<2$ so we need to consider only $\varepsilon<\frac{1}{2}$. Let $x \in \mathbb{R}^{n-r}$ be such that $\|C x\|=\|C\|$ and $\|x\|=1$. From (3.4) it follows that $A_{1}$ is nonsingular; hence there exists $y \in \mathbb{R}^{r}$ such that $\|y\|=1$ and $A_{1} y=\lambda C x$ for some $\lambda>0$. Therefore, $\left\|A_{1} y+C x\right\|=\left\|A_{1} y\right\|+\|C x\|$. Let $z=\left[\begin{array}{l}y \\ x\end{array}\right] \in \mathbb{R}^{n}$. Then $\|z\|^{2}=2$ and $A z=\left[\begin{array}{c}A_{1} y+C x \\ A_{2} x\end{array}\right]$. Therefore,

$$
\begin{aligned}
2(1+\varepsilon)^{2} & \geq\|A z\|^{2}=\left\|A_{1} y+C x\right\|^{2}+\left\|A_{2} x\right\|^{2} \\
& =\left(\left\|A_{1} y\right\|+\|C x\|\right)^{2}+\left\|A_{2} x\right\|^{2} \\
& \geq(1-\varepsilon)^{2}+((1-\varepsilon)+\|C\|)^{2} \\
& =2(1-\varepsilon)^{2}+2(1-\varepsilon)\|C\|+\|C\|^{2} .
\end{aligned}
$$

Solving this inequality for $\|C\|$, we obtain $\|C\| \leq 4 \varepsilon$.

Proof of Proposition 3.2 We first prove the proposition for $n=2^{m}$. For $k=$ $1,2, \ldots, n-1$ we fix $r=0,1, \ldots, m-1$ such that $2^{r} \leq k<2^{r+1}$ and define

$$
j_{k}=2^{m-r-1}\left(2\left(k-2^{r}\right)+1\right)+1 .
$$

Let $C_{k}$ be the matrix obtained from $A$ by setting to 0 all the coefficients except those at the intersections of columns $j_{k}, j_{k}+1, \ldots, j_{k}+2^{m-r-1}$ with rows $1,2, \ldots, i_{j_{k}}$. We still have $\left\|C_{k}\right\| \leq 4 \varepsilon$. 
Now let $D=\left[d_{i, j}\right]$ and $E=\left[e_{i, j}\right]$ be two matrices obtained from $A$ by setting some of the coefficients to 0 . We define $D \backslash E=\left[f_{i, j}\right]$ as the matrix obtained from $A$ by setting to 0 all coefficients except those which are nonzero in $D$ and equal to zero in $E$, i.e.,

$$
f_{i, j}= \begin{cases}a_{i, j} & \text { if } d_{i, j} \neq 0 \text { and } e_{i, j}=0, \\ 0 & \text { otherwise }\end{cases}
$$

For $r=0,1, \ldots, m-1$ we now define

$$
B_{r}=\left(\sum_{k=1}^{2^{r+1}-1} C_{k}\right) \backslash\left(\sum_{k=1}^{2^{r}-1} C_{k}\right)
$$

We show that $\left\|B_{r}\right\| \leq 4 \varepsilon$. Let $D_{l}=C_{l} \backslash\left(\sum_{k=1}^{l-1} C_{l}\right)$. Because $\left\|C_{l}\right\| \leq 4 \varepsilon$ and $D_{l}$ is obtained from $C_{l}$ by setting some rows to 0 , we have $\left\|D_{l}\right\| \leq 4 \varepsilon$. Observe that $B_{r}=\sum_{l=2^{r}}^{2^{r+1}-1} D_{l}$ and each of the matrices $D_{2^{r}}, D_{2^{r}+1}, \ldots, D_{2^{r+1}-1}$ has nonzero coefficients in different rows and columns. Hence

$$
\left\|B_{r}\right\| \leq \max \left(\left\|D_{2^{r}}\right\|,\left\|D_{2^{r}+1}\right\|, \ldots,\left\|D_{2^{r+1}-1}\right\|\right) \leq 4 \varepsilon
$$

Because $B=B_{0}+B_{1}+\cdots+B_{m-1}$ we get $\|B\| \leq m \cdot 4 \varepsilon=4 \varepsilon \cdot \log _{2} n$.

We deal with the situation when $n \neq 2^{m}$ in the following way: let $m=\left\lceil\log _{2} n\right\rceil$. We extend the matrix $A$ to a $2^{m} \times 2^{m}$ matrix $A^{\prime}=\left[a_{i, j}\right]_{i, j=1}^{2^{m}}$ by defining

$$
a_{i, j}= \begin{cases}1 & \text { for } n+1 \leq i=j \leq 2^{m} \\ 0 & \text { for } n+1 \leq i \leq 2^{m} \text { or } n+1 \leq j \leq 2^{m}\end{cases}
$$

For $j=n+1, \ldots, 2^{m}$ we define $i_{j}=j-1$. The matrix $A^{\prime}$ satisfies the assumptions of the lemma, and the matrix $B^{\prime}$ obtained from $A^{\prime}$ satisfies $\left\|B^{\prime}\right\| \leq 4 \varepsilon \cdot m$. Because $B$ is a sub-matrix of $B^{\prime}$, we have $\|B\| \leq\left\|B^{\prime}\right\| \leq 4 \varepsilon \cdot\left\lceil\log _{2} n\right\rceil$. The proof of the lemma is complete.

Proof of Theorem 3.1 We assume that $f_{k} \neq 0$. Otherwise $f_{S}=0$ as well and the inequality (3.1) is trivially satisfied.

For a given closed subspace $U \subset \mathcal{H}$ let $P_{U}$ be the orthogonal projection onto $U$. Let $\phi_{1}, \phi_{2}, \ldots, \phi_{S} \in \Phi$ be the distinct elements returned by the first $S$ iterations of the OMP when applied to $f$. For $U_{v}=\operatorname{span}\left(\phi_{1}, \ldots, \phi_{v}\right)$ and $k \leq v \leq S$ we have

$$
f_{v}=f-P_{U_{v}} f=f_{k}-P_{U_{v}} f_{k}
$$

and $\left\langle f_{k}, \phi_{j}\right\rangle=0$ for $j \in\{1, \ldots, k\}$.

For $f \in \mathcal{H}$ let

$$
d(f)=\sup _{g \in \Phi}|\langle f, g\rangle| .
$$


Let us fix $\psi \in U_{v}$ with $\|\psi\|=1$ and $\psi \perp U_{\nu-1}$. Then $\left\|f_{\nu-1}\right\|^{2}=\left\|f_{v}\right\|^{2}+$ $\left\langle f_{\nu-1}, \psi\right\rangle^{2}$. Since $d\left(f_{v-1}\right)=\left|\left\langle f_{\nu-1}, \phi_{\nu}\right\rangle\right|,\left\|\phi_{\nu}\right\| \leq 1+\varepsilon$ and $\left|\left\langle f_{\nu-1}, \psi\right\rangle\right| \geq$ $\left|\left\langle f_{v-1},\left\|\phi_{v}\right\|^{-1} \phi_{v}\right\rangle\right|$ we get

$$
\left\|f_{v}\right\|^{2} \leq\left\|f_{v-1}\right\|^{2}-(1+\varepsilon)^{-2} d\left(f_{\nu-1}\right)^{2} .
$$

Repeating this we obtain

$$
\left\|f_{S}\right\|^{2} \leq\left\|f_{k}\right\|^{2}-(1+\varepsilon)^{-2} \sum_{\nu=k+1}^{S} d\left(f_{\nu}\right)^{2}
$$

which implies

$$
\left\|f_{S}\right\|^{2} \leq 2\left\|f_{k}\right\|\left(\left\|f_{k}\right\|-(1+\varepsilon)^{-1}\left(\sum_{\nu=k+1}^{S} d\left(f_{\nu}\right)^{2}\right)^{1 / 2}\right)
$$

We will now provide a lower estimate for $\left(\sum_{v=k+1}^{S} d\left(f_{v}\right)^{2}\right)^{1 / 2}$.

Let $g_{1}, \ldots, g_{S-k} \in \Phi$ be distinct elements which have the biggest scalar products with $f_{k}$, i.e.,

$$
\left|\left\langle f_{k}, g_{1}\right\rangle\right| \geq\left|\left\langle f_{k}, g_{2}\right\rangle\right| \geq \cdots \geq\left|\left\langle f_{k}, g_{S-k}\right\rangle\right| \geq \sup \left\{\left|\left\langle f_{k}, g\right\rangle\right|: \phi \in \Phi, \phi \neq g_{i}\right\},
$$

and each $g_{i}, i \in\{1, \ldots, S-k\}$, is different from all $\phi_{j}, j \in\{1, \ldots, k\}$. Because $f_{k} \neq 0$, we have $d\left(f_{k}\right)=\left|\left\langle f_{k}, g_{1}\right\rangle\right|>0$. Observe also that $g_{1}=\phi_{k+1}$. We will also need a different enumeration of $g_{i}$ 's that will allow us to apply Proposition 3.2. To do this we show that there exists a bijective mapping $\pi:\{k+1, \ldots, S\} \rightarrow\{1, \ldots, S-k\}$ such that

$$
\text { if } g_{\pi(v)}=\phi_{j}, \quad \text { then } j>v \text { for } v=k, k+1, \ldots, S-1 \text {. }
$$

Let $A=\left\{g_{1}, \ldots, g_{S-k}\right\} \cap\left\{\phi_{k+1}, \ldots, \phi_{S-1}\right\}=\left\{\phi_{j_{1}}, \ldots, \phi_{j_{r}}\right\}$. We assume that

$$
k+1=j_{1}<j_{2}<\cdots<j_{r} .
$$

Define $\pi(k+\mu)=j_{\mu+1}$ for $\mu=0, \ldots, r-1$. The set $\left\{g_{1}, \ldots, g_{S-k}\right\} \backslash A$ is exhausted in an arbitrary way by $g_{\pi(k+r)}, \ldots, g_{\pi(S-1)}$. Now the property (3.7) follows from the fact that $g_{\pi(k)}=\phi_{k+1}$ and the ordering of $j_{1}, \ldots, j_{r}$.

By the definition of $d\left(f_{v}\right)$ we have $d\left(f_{v}\right) \geq\left|\left\langle f_{\nu}, g_{\pi(v)}\right\rangle\right|$, and by (3.5) $\left\langle f_{\nu}, g_{\pi(v)}\right\rangle=$ $\left\langle f_{k}, g_{\pi(v)}\right\rangle-\left\langle P_{U_{v}} f_{k}, g_{\pi(v)}\right\rangle$.

Let us define

$$
a_{v}=\overline{\left\langle f_{k}, g_{\pi(v)}\right\rangle} \cdot\left(\sum_{\nu=k+1}^{S}\left|\left\langle f_{k}, g_{\pi(v)}\right\rangle\right|^{2}\right)^{-1 / 2} .
$$


(Note that because $d\left(f_{k}\right)>0$, we have $\sum_{v=k+1}^{S}\left|\left\langle f_{k}, g_{\pi(v)}\right\rangle\right|^{2}>0$ as well.) Then $\sum_{v=k+1}^{S}\left|a_{v}\right|^{2}=1$, and

$$
\begin{aligned}
\left(\sum_{\nu=k}^{S-1} d\left(f_{\nu}\right)^{2}\right)^{1 / 2} & \geq\left(\sum_{\nu=k+1}^{S}\left|\left\langle f_{\nu}, g_{\pi(v)}\right\rangle\right|^{2}\right)^{1 / 2} \geq\left|\sum_{\nu=k+1}^{S} a_{\nu}\left\langle f_{\nu}, g_{\pi(v)}\right\rangle\right| \\
& \geq\left|\sum_{\nu=k+1}^{S} a_{\nu}\left\langle f_{k}, g_{\pi(v)}\right\rangle\right|-\left|\sum_{\nu=k+1}^{S} a_{\nu}\left\langle P_{U_{\nu}} f_{k}, g_{\pi(v)}\right\rangle\right| \\
& =\left(\sum_{i=1}^{S-k}\left|\left\langle f_{k}, g_{i}\right\rangle\right|^{2}\right)^{1 / 2}-\mid\left\langle f_{k}, \sum_{\nu=k+1}^{S} a_{\nu} P_{U_{\nu}} g_{\pi(v)}\right\rangle .
\end{aligned}
$$

We now estimate

$$
\left|\left\langle f_{k}, \sum_{\nu=k+1}^{S} a_{\nu} P_{U_{\nu}} g_{\pi(\nu)}\right\rangle\right| \leq\left\|f_{k}\right\|\left\|\sum_{\nu=k+1}^{S} a_{\nu} P_{U_{\nu}} g_{\pi(v)}\right\| .
$$

Now let us consider the system of vectors from $\mathcal{H}$,

$$
\phi_{1}, \ldots, \phi_{S}, g_{\pi(r+1)}, \ldots, g_{\pi(S-k)},
$$

arranged in this particular order. Since this system consists of elements from $\Phi$ we will denote it as $\left\{\phi_{j}\right\}_{j=1}^{R}$ with $R=2 S-k-r<2 S$. Let $\rho(v)$ be such that $g_{\pi(v)}=$ $\phi_{\rho(v)}$ for $v=l+1, \ldots, S$. Observe that the mapping $v \mapsto \rho(v)$ is increasing and $\rho(v)>v$.

Now let $\psi_{1}, \ldots, \psi_{R}$ be the Gram-Schmidt orthonormalization of the system (3.10). Then

$$
\phi_{j}=\sum_{i=1}^{j} t_{i, j} \psi_{i}
$$

and the upper-triangular $R \times R$ matrix $T=\left[t_{i, j}\right]$ satisfies the assumptions of Proposition 3.2, which follows from the RIP of the dictionary $\Phi$.

Note that we have

$$
P_{U_{v}} g_{\pi(v)}=P_{U_{v}} \phi_{\rho(v)}=\sum_{i=1}^{v} t_{i, \rho(v)} \psi_{i}
$$

For each column index $j \in\{1,2, \ldots, R\}$ we define a row index $i_{j}$ so that $i_{\rho(v)}=v$, and for $j \notin\{\rho(k+1), \ldots, \rho(S)\}$ we choose $i_{j}$ so that the sequence $\left(i_{j}\right)_{j=1}^{R}$ is nondecreasing and $i_{j}>i$. Let the matrix $\tilde{B}=\left[b_{i, j}\right]$ with $i, j=1, \ldots, R$ be defined as

$$
b_{i, j}= \begin{cases}t_{i, j} & \text { if } 1 \leq i \leq i_{j} \\ 0 & \text { otherwise }\end{cases}
$$


By Proposition 3.2

$$
\|\tilde{B}\| \leq 4 \varepsilon \cdot\left\lceil\log _{2} R\right\rceil
$$

Let $B_{j}$ denote the $i$ th column of the matrix $\tilde{B}$ and set

$$
B=\left[B_{\rho(k+1)}, \ldots, B_{\rho(S)}\right]
$$

Observe that

$$
\|B\| \leq\|\tilde{B}\| \leq 4 \varepsilon \cdot\left\lceil\log _{2} R\right\rceil \leq 4 \varepsilon \cdot\left\lceil 1+\log _{2} S\right\rceil .
$$

For the vector $a=\left[a_{k+1}, \ldots, a_{S}\right]^{T}$ (defined in (3.8)) we have $\|a\|=1$ and

$$
\left\|\sum_{\nu=k+1}^{S} a_{\nu} P_{U_{\nu}} g_{\pi(v)}\right\|=\|B a\| \leq\|B\|\|a\| \leq 4 \varepsilon \cdot\left\lceil\log _{2}(2 S-k)\right\rceil .
$$

Next we estimate the term $\left(\sum_{i=1}^{S-k}\left|\left\langle f_{k}, g_{i}\right\rangle\right|^{2}\right)^{1 / 2}$. Let $\eta_{1}, \ldots, \eta_{S-k} \in \Phi$ be distinct elements such that for $V=\operatorname{span}\left(\eta_{1}, \ldots, \eta_{S-k}\right)$ we have

$$
\sigma_{S-k}\left(f_{k}\right)=\left\|f_{k}-P_{V} f_{k}\right\| \text {. }
$$

Let the scalars $b_{1}, \ldots, b_{S-k}$ be such that

$$
P_{V} f_{k}=\sum_{j=1}^{S-k} b_{j} \eta_{j}
$$

Observe that $\left\|P_{V} f_{k}\right\| \geq\left\|f_{k}\right\|-\sigma_{S-k}\left(f_{k}\right)$, which combined with the RIP gives us

$$
\left(\sum_{j=1}^{S-k}\left|b_{j}\right|^{2}\right)^{1 / 2} \geq \frac{1}{1+\varepsilon}\left(\left\|f_{k}\right\|-\sigma_{S-k}\left(f_{k}\right)\right) .
$$

Using Proposition 2.1 and the RIP we next obtain

$$
\begin{aligned}
\left(\sum_{j=1}^{S-k}\left\langle f_{k}, \eta_{j}\right\rangle\right)^{1 / 2} & =\left(\sum_{j=1}^{S-k}\left\langle P_{V} f_{k}, \eta_{j}\right\rangle\right)^{1 / 2} \\
& \geq(1-\varepsilon)\left\|P_{V} f_{k}\right\| \\
& \geq(1-\varepsilon)^{2}\left(\sum_{j=1}^{S-k}\left|b_{j}\right|^{2}\right)^{1 / 2} .
\end{aligned}
$$

From (3.13) and (3.14) we get

$$
\left(\sum_{i=1}^{S-k}\left|\left\langle f_{k}, g_{i}\right\rangle\right|^{2}\right)^{1 / 2} \geq\left(\sum_{j=1}^{S-k}\left|\left\langle f_{k}, \eta_{j}\right\rangle\right|^{2}\right)^{1 / 2} \geq \frac{(1-\varepsilon)^{2}}{1+\varepsilon}\left(\left\|f_{k}\right\|-\sigma_{S-k}\left(f_{k}\right)\right) .
$$


From (3.6), (3.9), (3.15), (3.11), and (3.12) we obtain

$$
\begin{aligned}
\left\|f_{S}\right\|^{2} \leq & 2\left\|f_{k}\right\|\left(\left(\frac{1-\varepsilon}{1+\varepsilon}\right)^{2} \sigma_{S-k}\left(f_{k}\right)\right. \\
& \left.+\left(1-\left(\frac{1-\varepsilon}{1+\varepsilon}\right)^{2}+4 \varepsilon\left\lceil 1+\log _{2} S\right\rceil\right)\left\|f_{k}\right\|\right) \\
\leq & 2\left\|f_{k}\right\|\left(\sigma_{S-k}\left(f_{k}\right)+4 \varepsilon\left(2+\left\lceil\log _{2} S\right\rceil\right)\left\|f_{k}\right\|\right) .
\end{aligned}
$$

The proof is complete.

For dictionaries with positive coherence, J. Tropp [14], slightly improving the estimate from [8], showed:

Theorem 3.4 If the dictionary $\Phi$ has coherence $\eta=\eta(\Phi)>0$, then

$$
\left\|f_{m}\right\| \leq \sqrt{1+6 m} \sigma_{m}(f)
$$

for $m<(3 \eta)^{-1}$.

Using the above theorem we obtain:

Theorem 3.5 Assume that the dictionary $\Phi$ satisfies $\operatorname{HRIP}(k, \delta)$. Then there exists a constant $C_{\delta}$ such that for $m \leq \sqrt{k}(6 \delta)^{-1}$ we have

$$
\left\|f_{m\left\lceil 4 \log _{2} m-1\right\rceil}\right\| \leq C_{\delta} \sigma_{m}(f) .
$$

Proof By HRIP and Proposition 2.2 the dictionary $\Phi$ has coherence

$$
\eta \leq \frac{3 \delta}{\sqrt{k}} .
$$

We take

$$
m \leq \frac{1}{6} \delta^{-1} k^{1 / 2}
$$

so that (3.16) holds. We define $m_{l}:=m\left(2^{l}-1\right)$ for $l=1,2, \ldots$ Let us fix $S=a k^{\gamma}$, where $\gamma \in\left(\frac{1}{2}, \frac{3}{4}\right)$ and $a \in(0,1)$ is chosen so that $S$ is sufficiently large and integer. By HRIP the dictionary $\Phi$ satisfies $\operatorname{RIP}(2 S, \varepsilon)$ with

$$
\varepsilon=a^{\frac{1}{2}} \delta k^{-\frac{1-\gamma}{2}} \text {. }
$$

The following is true:

Lemma 3.6 There exists a constant $B=B(\delta, a, \gamma)$ such that

$$
4 \varepsilon\left(2+\left\lceil\log _{2} S\right\rceil\right) \leq B m^{-1 / 4} .
$$


We can take

$$
B(\delta, a, \gamma) \leq e \cdot 2^{\frac{5}{4}+\frac{3}{8 \gamma}} \cdot 3^{-\frac{1}{4}} \cdot a^{\frac{1}{2}} \cdot\left(2+\frac{8 \gamma}{(4 \gamma-3) \ln 2}\right) \delta^{\frac{3}{4}} .
$$

The proof of this lemma is presented later.

Using Theorem 3.1, inequality (3.19), and the fact that $\sigma_{n}\left(f_{k}\right) \leq \sigma_{n-k}(f)$ for $k \leq n$ we get

$$
\left\|f_{m_{l}}\right\|^{2} \leq 2\left\|f_{m_{l-1}}\right\|\left(\sigma_{m}(f)+B m^{-1 / 4}\left\|f_{m_{l-1}}\right\|\right)
$$

as long as $m_{l} \leq S$.

From (3.16) we have $\left\|f_{m}\right\| \leq \sqrt{7} m^{1 / 2} \sigma_{m}(f)$. If we know that for some constant $D_{l-1}$ we have $\left\|f_{m_{l-1}}\right\| \leq D_{l-1} m^{\gamma} \sigma_{m}(f)$ for $\gamma \geq \frac{1}{4}$, from (3.20) using inequality $\sqrt{1+z} \leq 2 \sqrt{z}$ for $z \geq 1$ we obtain

$$
\left\|f_{m_{l}}\right\| \leq 2 D_{l-1} B^{1 / 2} m^{\gamma-\frac{1}{8}} \sigma_{m}(f) .
$$

Let $D_{1}=\sqrt{7}$, so that $(1+6 m)^{1 / 2} \leq D_{1} m^{1 / 2}$. From (3.16) and (3.21) we obtain (iteratively for $l=2,3,4$ )

$$
\left\|f_{m_{4}}\right\| \leq 8 D_{1} B^{3 / 2} m^{1 / 8} \sigma_{m}(f) .
$$

Set $D_{4}=8 D_{1} B^{3 / 2}$.

If $m^{1 / 8}<4 B D_{4}$, then

$$
\left\|f_{m_{4}}\right\| \leq 4 B D_{4}^{2} \sigma_{m}(f)
$$

which ends the proof, yielding $C_{\delta} \geq 4 B D_{4}^{2}$.

If $m^{1 / 8}>4 B D_{4}$, then (3.22) becomes

$$
\left\|f_{m_{4}}\right\| \leq D_{4} m^{1 / 8} \sigma_{m}(f),
$$

and we inductively show

$$
\left\|f_{m_{l}}\right\| \leq D_{l} m^{2^{-l+1}} \sigma_{m}(f)
$$

with $D_{l} \leq 4 D_{4}$. Assume that (3.23) holds for some $l \geq 4$. From (3.20) we have

$$
\begin{aligned}
\left\|f_{m_{l+1}}\right\|^{2} & \leq 2 D_{l} m^{2^{-l+1}}\left(1+B D_{l} m^{-\frac{1}{4}+2^{-l+1}}\right) \sigma_{m}(f)^{2} \\
& \leq 2 D_{l} m^{2^{-l+1}}\left(1+4 B D_{4} m^{-\frac{1}{8}}\right) \sigma_{m}(f)^{2} \\
& \leq 4 D_{l} m^{2^{-l+1}} \sigma_{m}(f)^{2} .
\end{aligned}
$$

Hence $\left\|f_{m+l+1}\right\| \leq 2 D_{l}^{1 / 2} m^{2^{-l}} \sigma_{m}(f)=D_{l+1} m^{2^{-l}} \sigma_{m}(f)$ and $D_{l+1} \leq 2 D_{l}^{1 / 2} \leq$ $2\left(4 D_{4}\right)^{1 / 2} \leq 4 D_{4}$. 
We now take $l=l^{*}$ such that $m^{2^{-l+1}} \leq 2$. A routine calculation shows that it suffices to take $l^{*}=\left\lceil\log _{2} \log _{2} m\right\rceil+1$. We then have

$$
\left\|f_{m\left\lceil 4 \log _{2} m-1\right\rceil}\right\| \leq\left\|f_{m_{l^{*}}}\right\| \leq 8 D_{4} \sigma_{m}(f) .
$$

Hence, if (3.17) holds, we can take $C_{\delta}=8 D_{4}=64 \cdot 7 \cdot B(\delta, a, \gamma)^{3 / 2}$.

Clearly, the constants we got in the above argument are far from being optimal.

Proof of Lemma 3.6 By (3.17) we have $m^{-1 / 4} \geq 6^{1 / 4} k^{-1 / 8} \delta^{1 / 4}$. Because $S=a k^{\gamma}$ and $\varepsilon$ is given by (3.18), we need

$$
B \geq 2^{\frac{7}{4}} 3^{-\frac{1}{4}} a^{\frac{1}{2}} \delta^{3 / 4} k^{1 / 8}\left(2+\left\lceil\log _{2} S\right\rceil\right) .
$$

A routine calculation shows that

$$
2+\left\lceil\log _{2} S\right\rceil \leq 3+\gamma \log _{2} k .
$$

Hence, it suffices that $B=2^{7 / 4} 3^{-1 / 4} \delta^{3 / 4} \cdot \sup _{k>0} h(k)$, with

$$
h(k)=k^{-\frac{3}{8}+\frac{\gamma}{2}}\left(3+\gamma \log _{2} k\right), \quad k>0 .
$$

The function $h$ has the maximum value of

$$
e \cdot 2^{-\frac{1}{2}+\frac{3}{8 \gamma}}\left(2+\frac{8 \gamma}{(4 \gamma-3) \ln 2}\right)
$$

\section{Closing Remarks}

Our results are a contribution to the general problem of comparing $\left\|f_{n}\right\|=$ $\left\|f-\mathrm{OMP}_{n} f\right\|$ with $\sigma_{n}(f)$. There are two main types of inequalities one may seek. One is the inequality of the form

$$
\left\|f_{m}\right\| \leq C_{m} \sigma_{m}(f)
$$

where we want the constant $C_{m}$ to be small, preferably independent of $m$. Another one is the inequality of the form

$$
\left\|f_{\eta(m)}\right\| \leq C \sigma_{m}(f)
$$

where $\eta(m)$ is a certain function of $m$, preferably not much bigger than $m$. Clearly the combination of both types is possible. An important factor in such inequalities is the range of $m$ 's for which they are valid. Our Theorem 3.1 (and Theorem 1.3 from [7]) provide tools to pass from inequality (4.1) to inequality (4.2) with $\eta(m) \sim\lfloor m \log m\rfloor$.

The main drawback of Theorem 3.5 is the restriction $m \leq c / \sqrt{k}$. The inspection of the proof shows that it is caused by an analogous restriction in Theorem 3.4. It is rather unlikely that the range of applicability of this theorem can be significantly 
improved, as it uses only the coherence of the dictionary. On the other hand, the value $\sqrt{1+6 m}$ which appears in Theorem 3.4 does not seem to be very essential. Replacing it by $m$ to any fixed power would be sufficient for our argument to work. Thus it seems to be an interesting problem to establish an analogue of Theorem 3.4 for dictionaries with HRIP. Let us state it as a conjecture:

Conjecture 1 Assume that the dictionary satisfies $\operatorname{HRIP}(k, \delta)$. There exist constants $C, c, \alpha$ and $\beta$ (possibly depending on $\delta$ ) such that for every $f$ and for $m \log ^{\alpha} m \leq c k$ we have

$$
\left\|f_{\left\lfloor m \log ^{\alpha} m\right\rfloor}\right\| \leq C m^{\beta} \sigma_{m}(f) .
$$

It would be especially interesting to have a constant in place of $\log ^{\alpha} m$. This however may require some restrictions on $m$. We have the following example to support this claim.

Let us fix integers $n>2$ and $s \leq n$ and set $k=n+s$. We fix a number $\beta$ with $\sqrt{\frac{n}{n-1}}<\beta<\sqrt{\frac{n+1}{n-1}}$ and define $x=n^{-1 / 2} \sum_{j=1}^{n} e_{j}$ and $\psi_{j}=e_{n+j}+\frac{\beta}{\sqrt{n}} x$ for $j=$ $1, \ldots, s$. We consider the dictionary in $\mathbb{R}^{k}$ given by Psi $=\left\{e_{j}\right\}_{j=1}^{n} \cup\left\{\left\|\psi_{j}\right\|^{-1} \psi_{j}\right\}_{j=1}^{s}$.

Proposition 4.1 The dictionary $\Psi$ described above has the following properties:

(i) OMP applied to $x$ first chooses all the $\psi_{j}$ 's and only later the $e_{j}$ 's.

(ii) The dictionary $\Psi$ has coherence $\eta(\Psi)<\beta n^{-1 / 2}$.

(iii) If $s \leq \beta^{-2} n$, then $\Psi$ has $\operatorname{RIP}(k, \epsilon)$ with $\epsilon=\beta \sqrt{\frac{s}{n}}+\frac{\beta^{2}}{n}$.

Proof $^{1}$ We analyze how OMP acts on vector $x$. Clearly $\left\langle x, e_{j}\right\rangle=\frac{1}{\sqrt{n}}$ and $\left\langle x, \psi_{j}\right\rangle=\frac{\beta}{\sqrt{n}}$. Note that $\left\|\psi_{j}\right\|=\left(1+\beta^{2} n^{-1}\right)^{1 / 2}$. Since $\beta\left(1+\beta^{2} n^{-1}\right)^{-1 / 2}>1$ we infer that in the first step OMP can choose any vector $\psi_{j}$, say $\psi_{1}$. Now assume that in the first $l$ steps OMP has chosen only $\psi_{j}$ 's, say $\psi_{1}, \ldots, \psi_{l}$. Clearly

$$
\operatorname{OMP}_{l}(x)=\sum_{j=1}^{l} a_{j} \psi_{j}=\sum_{j=1}^{l} a_{j} e_{n+j}+\gamma x
$$

with $\gamma=\frac{\beta}{\sqrt{n}} \sum_{j=1}^{l} a_{j}$. Since $\left\langle\mathrm{OMP}_{l}(x), \psi_{j}\right\rangle=\left\langle x, \psi_{j}\right\rangle$ for $j=1,2, \ldots, l$ we get $a_{j}+\gamma \frac{\beta}{\sqrt{n}}=\frac{\beta}{\sqrt{n}}$. Solving those equations we get

$$
\gamma=\frac{l \beta^{2}}{n+l \beta^{2}} \quad \text { and } \quad a_{j}=\frac{\beta \sqrt{n}}{n+l \beta^{2}} \quad \text { for } j=1,2, \ldots, l .
$$

\footnotetext{
${ }^{1}$ We would like to thank K. Cwalina for his remarks that simplified the proof.
} 
From (4.3) and (4.4) we get

$$
r_{l}=-\frac{\beta \sqrt{n}}{n+l \beta^{2}} \sum_{j=1}^{l} e_{n+j}+\frac{n}{n+\beta^{2} l} x .
$$

Now we can see what OMP does in the $(l+1)$-th step. For $j \leq n$ we have

$$
\left\langle r_{l}, e_{j}\right\rangle=\frac{\sqrt{n}}{n+\beta^{2} l},
$$

while for $j>l$ we have

$$
\left\langle r_{l},\left\|\psi_{j}\right\|^{-1} \psi_{j}\right\rangle=\left(1+\frac{\beta^{2}}{n}\right)^{-1 / 2} \frac{\beta \sqrt{n}}{n+\beta^{2} l} .
$$

Since $\left(1+\frac{\beta^{2}}{n}\right)^{-1 / 2} \beta>1$ we see that OMP chooses another $\psi_{j}$. This shows (i). To see (ii) we calculate the scalar products. Since all $\psi_{j}$ 's have the same norm we put $\mu=\left\|\psi_{j}\right\|^{-1}<1$. To see (iii) we write

$$
\begin{aligned}
\left\|\sum_{j=1}^{n} a_{j} e_{j}+\sum_{j=1}^{s} b_{j} \mu \psi_{j}\right\| & =\left\|\sum_{j=1}^{n} a_{j} e_{j}+\mu \sum_{j=1}^{s} b_{j} e_{n+j}+\frac{\beta \mu}{\sqrt{n}}\left(\sum_{j=1}^{s} b_{j}\right) \cdot x\right\| \\
& \leq \sqrt{\sum_{j=1}^{n} a_{j}^{2}+\sum_{j=1}^{s} b_{j}^{2}+\frac{\beta}{\sqrt{n}} \sum_{j=1}^{s}\left|b_{j}\right|} \\
& \leq \sqrt{\sum_{j=1}^{n} a_{j}^{2}+\sum_{j=1}^{s} b_{j}^{2}+\sqrt{s} \frac{\beta}{\sqrt{n}} \sqrt{\sum_{j=1}^{s}\left|b_{j}\right|^{2}}} \\
& \leq\left(1+\sqrt{s} \frac{\beta}{\sqrt{n}}\right) \sqrt{\sum_{j=1}^{n} a_{j}^{2}+\sum_{j=1}^{s} b_{j}^{2}} .
\end{aligned}
$$

Analogously we get

$$
\left\|\sum_{j=1}^{n} a_{j} e_{j}+\sum_{j=1}^{s} b_{j} \mu \psi_{j}\right\| \geq \mu\left(1-\sqrt{s} \frac{\beta}{\sqrt{n}}\right) \sqrt{\sum_{j=1}^{n} a_{j}^{2}+\sum_{j=1}^{s} b_{j}^{2}} .
$$

Since $\mu\left(1-\sqrt{s} \frac{\beta}{\sqrt{n}}\right) \geq 1-(1-\mu)-\sqrt{s} \frac{\beta}{\sqrt{n}}$ and $1-\mu<\beta^{2} n^{-1}$, we get (iii).

Open Access This article is distributed under the terms of the Creative Commons Attribution Noncommercial License which permits any noncommercial use, distribution, and reproduction in any medium, provided the original author(s) and source are credited.

\section{References}

1. Baraniuk, R., Davenport, M., DeVore, R., Wakin, M.: A simple proof of the restricted isometry property for random matrices. Constr. Approx. 28(3), 253-263 (2008) 
2. Candés, E.J., Tao, T.: Decoding by linear programming. IEEE Trans. Inf. Theory 51(12), 4203-4215 (2005)

3. Christensen, O.: An Introduction to Frames and Riesz Bases. Applied and Numerical Harmonic Analysis. Birkhäuser, Boston (2003)

4. Cohen, A., Dahmen, W., DeVore, R.: Compressed sensing and best $k$-term approximation. J. Am. Math. Soc. 22(1), 211-231 (2009)

5. Davenport, M.A., Wakin, M.B.: Analysis of orthogonal matching pursuit using the restricted isometry property. Preprint (2009)

6. Donoho, D.L.: Compressed sensing. IEEE Trans. Inf. Theory 52(4), 1289-1306 (2006)

7. Donoho, D.L., Elad, M., Temlyakov, V.N.: On Lebesgue-type inequalities for greedy approximation. J. Approx. Theory 147(2), 185-195 (2007)

8. Gilbert, A.C., Muthukrishnan, S., Strauss, M.J.: Approximation of functions over redundant dictionaries using coherence. In: Proceedings of the Fourteenth Annual ACM-SIAM Symposium on Discrete Algorithms, Baltimore, MD, 2003, pp. 243-252. ACM, New York (2003)

9. Huber, P.J.: Projection pursuit. Ann. Stat. 13(2), 435-525 (1985). With discussion

10. Jones, L.K.: On a conjecture of Huber concerning the convergence of projection pursuit regression. Ann. Stat. 15(2), 880-882 (1987)

11. Kwapien, S., Pelczyński, A.: The main triangle projection in matrix spaces and its applications. Studia Math. 34, 43-68 (1970)

12. Mendelson, S., Pajor, A., Tomczak-Jaegermann, N.: Uniform uncertainty principle for Bernoulli and subgaussian ensembles. Constr. Approx. 28(3), 277-289 (2008)

13. Temlyakov, V.N.: Greedy approximation. Ada Numer. 17, 235-409 (2008)

14. Tropp, J.A.: Greed is good: algorithmic results for sparse approximation. IEEE Trans. Inf. Theory 50(10), 2231-2242 (2004) 